\title{
Endogenous Rhythms in Period1 Mutant Suprachiasmatic Nuclei In Vitro Do Not Represent Circadian Behavior
}

\author{
Julie S. Pendergast, Rio C. Friday, and Shin Yamazaki \\ Department of Biological Sciences, Vanderbilt University, Nashville, Tennessee, 37235-1634
}

The mammalian circadian pacemaker in the suprachiasmatic nuclei (SCN) controls daily rhythms of behavior and physiology. Lesions of the SCN cause arrhythmicity of locomotor activity, and transplants of fetal SCN tissue restore rhythmic behavior that is consistent with the periodicity of the donor's genotype, suggesting that the SCN determines the period of the circadian behavioral rhythm. While several studies have demonstrated that the circadian characteristics of in vitro SCN rhythms represent circadian behavior, others have shown that the periods of explanted SCN are not always congruent with locomotor activity. We find that the aberrant rhythms of ex vivo SCN lacking functional Period1 $\left(\right.$ Per1 $^{-1-}$ ) do not represent the behavioral rhythms of the mutant animals. Surprisingly, in C57BL/6J Per1 ${ }^{-1-}$ mice, the real-time circadian gene promoter activity rhythm is weak or absent in adult SCN slices in vitro even though the free-running wheel-running activity rhythm is indistinguishable from wild-type $\left(\mathrm{Perl}^{+/+}\right)$mice. While some neurons in Per1 ${ }^{-/-}$SCN explants exhibit robust circadian rhythms, others have irregular and/or low-amplitude rhythms. Together, these data suggest that either a small population of rhythmic neurons in the Per ${ }^{-/-} \mathrm{SCN}$ is sufficient to control wheel-running activity or that in vivo physiological factors can compensate for the aberrant endogenous rhythms of $\mathrm{Per}^{-1-}$ SCN.

\section{Introduction}

Circadian rhythms are self-sustained oscillations in physiology and behavior with endogenous periods of $\sim 24 \mathrm{~h}$ that can be entrained to environmental cues such as the light/dark cycle or temperature (Takahashi et al., 2001). In mammals, the master circadian clock, located in the suprachiasmatic nuclei (SCN) of the anterior hypothalamus, plays a critical role in the generation of daily rhythms. Transplanting an SCN graft into an SCNlesioned hamster or mouse restores its locomotor activity rhythm with a period consistent with the donor animal's genotype, suggesting that the SCN controls the period of the circadian behavioral rhythm (Ralph et al., 1990; Silver et al., 1996; Sujino et al., 2003). In addition, averaging the period of the firing rhythms of individual dispersed SCN neurons from heterozygous and homozygous tau mutant hamsters matches the behavioral periods of the whole animals (Liu et al., 1997). Similarly, the lengthened behavioral period of locomotor activity in Clock mutant $(\Delta 19)$ heterozygote mice matches the period of the spontaneous firing rate in dispersed SCN neurons and the period of circadian promoter-driven reporter expression in SCN explants from these mice (Herzog et al., 1998; Yoo et al., 2005). In contrast, other studies have shown that the period of the SCN and of locomotor activity may not always be congruent. For example, mice exposed to non-24 $\mathrm{h}$ T-cycles exhibit aftereffects, or period changes, in

\footnotetext{
Received July 8, 2009; revised 0ct. 7, 2009; accepted 0ct. 10, 2009.

This research was supported by a National Institutes of Health grant (NS051278 to S.Y.) and by the Vanderbilt University Summer Research Program (to R.C.F.). We thank David Weaver for $\mathrm{mPer} 1^{1 / d{ }^{-/-}}$mice and Akiko Hida and Hajime Tei for Per1-luc mice.

Correspondence should be addressed to Shin Yamazaki, Department of Biological Sciences, Vanderbilt University, VU Station B, Box 35-1634, Nashville, TN 37235. E-mail: shin.yamazaki@vanderbilt.edu.

D0I:10.1523/JNEUROSCI.3261-09.2009

Copyright $\odot 2009$ Society for Neuroscience ～0270-6474/09/2914681-06\$15.00/0
}

locomotor behavior and in the circadian gene expression rhythm in cultured SCN (Aton et al., 2004; Molyneux et al., 2008). However, the aftereffect periods of the SCN in vitro and of behavior are negatively correlated. Interestingly, a previous study found that even though individual, dispersed cells of circadian mutant SCN may not reflect the behavioral phenotype of the corresponding mutant mice, rhythms in whole SCN explants correlated strongly with circadian behavior (Liu et al., 2007).

The molecular mechanism of endogenous rhythm generation in the SCN is modeled as interlocking transcriptional and translational feedback loops of circadian gene expression (Ko and Takahashi, 2006). Two Period homologs (Per1 and Per2) are known to be negative components of the feedback loops. The functions of some circadian genes have been investigated by generating circadian gene knock-out mice, including mice with nonfunctional Per1 (Bae et al., 2001; Cermakian et al., 2001; Zheng et al., 2001). Unlike previous studies of $P e r 1^{-1-}$ mice on mixed genetic backgrounds (Cermakian et al., 2001; Zheng et al., 2001), in the current study we report that the periods of circadian behavior in Per1 ${ }^{-1-}$ mice congenic with the C57BL/6J strain are similar to $\mathrm{Per} \mathrm{I}^{+/+}$mice. Surprisingly, though, we find that the endogenous rhythms in Per1 ${ }^{-1-}$ SCN explants are not congruent with the behavioral phenotypes of the mice, suggesting that in vivo factors may compensate for aberrant rhythms in mutant SCN.

\section{Materials and Methods}

Animals. mPer1 ${ }^{l d c-l-}$ mice (Bae et al., 2001) (provided by Dr. David Weaver, University of Massachusetts, Worcester, MA) (congenic with the 129/sv genetic background) were backcrossed with male and female wild-type C57BL/6J mice (The Jackson Laboratory) for 10-11 generations $\left(\mathrm{C} 57 \mathrm{BL} / 6 \mathrm{~J} \mathrm{Per1}^{-1-}\right.$ mice are available from The Jackson Laboratory, stock \#10491). Per1 ${ }^{+/-}$mice were then crossed with C57BL/6J 
Per1-luc transgenic mice (1-8L) (Herzog et al., 2004) to generate mice that were heterozygous for the Perl gene and for the Per1-luc transgene. Perl ${ }^{+/-}$mice were then crossed with $\operatorname{Perl}^{+/-}$mice with the Per1-luc transgene to generate $\mathrm{Perl}^{+/+}, \mathrm{Per}^{+/-}$, and $\mathrm{Perl}^{-/-}$mutant mice that expressed Per1-luc (N10 to N11) that were used for experiments. To assess the expression of the PERIOD2::LUCIFERASE fusion protein, Perl ${ }^{+/+}$and Perl ${ }^{-/-}$mice were generated as described above except that $\mathrm{Per}^{+/-}$mice were crossed with C57BL/6J PER2::LUC mice (Yoo et al., 2004). Genotype was determined by PCR amplification of tail DNA as previously described (Bae et al., 2001). The mice were bred and group housed in the Vanderbilt University animal facility in a $12 \mathrm{~h} \mathrm{light} / 12 \mathrm{~h}$ dark cycle [12L:12D (LD)] and provided food and water ad libitum. Male and female mice, aged 25-204 d (mean \pm SD: $65.2 \pm 38.4 \mathrm{~d}$ ) at the beginning of the experiment, were used for assessing behavior and for preparing tissue explants. All experiments were conducted in accordance with the guidelines of the Institutional Animal Care and Use Committee at Vanderbilt University.

Analysis of wheel-running activity. For experiments assessing wheelrunning activity, mice were singly housed in cages $(33 \times 17 \times 14 \mathrm{~cm})$ with unlimited access to a running wheel (diameter: $11 \mathrm{~cm}$ ), food, and water. The cages were placed in light-tight, ventilated boxes where the light intensity was 350 lux. Wheel running activity was monitored by a micro-switch-activated signal using the ClockLab system (Actimetrics) and was collected by computer every minute. Analysis was performed using ClockLab software. Free-running period was determined by using a $\chi^{2}$ periodogram for $15 \mathrm{~d}$ [days $1-15$ in constant darkness (DD)]. The amplitude $\left(Q_{p}\right)$ of the wheel-running rhythm was the peak value of the $\chi^{2}$ periodogram. Total activity level was determined by counting the total number of wheel revolutions from days 1-15 in DD and then averaging them to determine daily activity level. The phase angle of entrainment was defined as the time difference between lights off and the predicted time of activity onset on the first day in DD. This was calculated by drawing a regression line to activity onset for days $1-5$ in DD and then extending the regression line to the last day in LD. A negative phase angle was obtained when activity started before lights off and a positive phase angle was obtained when activity started after lights off.

Luminescence recording and imaging. The detailed methods for realtime measurement of luminescence from ex vivo tissues have been described (Yamazaki and Takahashi, 2005). Coronal slices of the SCN (300 $\mu \mathrm{m}$ ) were prepared by trimming away most extra-SCN tissue (small and middle sizes shown in supplemental Fig. S3C, available at www.jneurosci. org as supplemental material) and bioluminescence was measured using the LumiCycle apparatus (Actimetrics). Bioluminescence imaging was performed using a water-cooled CCD camera (ORCAIIERW, Hamamatsu). The SCN explant was placed on an $x-y-z$ stage in a temperaturecontrolled dark box, and the image was processed through direct coupling of a non-infinity-corrected microscope objective (NPLM20X, Union Optical) to the camera with a $3-\mathrm{cm}$-long extension tube. Focus was adjusted by moving the motorized $z$-stage controlled from outside of the box. Twenty-nine-minute exposures were collected every $30 \mathrm{~min}$. The images were analyzed for signal intensity using ImageJ software (National Institutes of Health). LumiCycle software (Actimetrics) was used to subtract the $24 \mathrm{~h}$ moving average from the raw luminescence data, to smooth the data by $0.5 \mathrm{~h}$ adjacent averaging, and to determine the amplitude of the first cycle of the baseline-subtracted, smoothed data. To determine period and phase, the baseline-subtracted and smoothed data were exported to ClockLab (Actimetrics). The period was determined by fitting a regression line to the acrophase of at least $3 \mathrm{~d}$ of the Perl-luc rhythm, and the phase was determined from the first peak of Perl-luc expression in vitro.

Statistical analysis. Statistical analysis was performed using SigmaStat (Systat Software). One-way ANOVA followed by post hoc Fisher's least significant difference (LSD) tests was used for comparison of more than two groups, and independent $t$ tests (two-tailed) were used to compare two groups except when data were not normally distributed or variances were not homogeneous. The Kolmogorov-Smirnov test (with Lilliefors' correction) was used to test data for normality. For nonparametric analyses, the Kruskal-Wallis one-way ANOVA on ranks followed by post hoc Dunn's method was used. Significance was ascribed at $p<0.05$.

\section{Results}

\section{Circadian wheel-running behavior of $\mathrm{Per} \mathrm{1}^{-/-}$mice congenic} with the $\mathrm{C} 57 \mathrm{BL} / 6 \mathrm{~J}$ strain is similar to $\mathrm{PerI}^{+/+}$mice

Previous studies have demonstrated that Per $1^{-1-}$ mice on a mixed genetic background have shortened free-running periods (Cermakian et al., 2001; Zheng et al., 2001) and those isogenic with the 129/sv strain sometimes become arrhythmic in DD (Bae et al., 2001). To determine whether these phenotypes were due to the genetic background of the mice, we generated Per $1^{-1-}$ mice congenic with the C57BL/6J strain by backcrossing mPer $1^{l d c-1-}$ mutants (Bae et al., 2001) for 10-11 generations with C57BL/6J wild-type mice (The Jackson Laboratory). We found that the free-running periods of $\mathrm{C} 57 \mathrm{BL} / 6 \mathrm{~J} \mathrm{Per1}{ }^{-1-}$ mice did not differ from $\operatorname{Per} 1^{+/+}$and $\operatorname{Per} 1^{+/-}$mice $\left(F_{(2,26)}=0.98, p=0.39\right)$ (Fig. $1 A-D)$. The phase angle of entrainment was similar between Per1 ${ }^{+/+}, \operatorname{Per}^{+/-}$, and Per1 ${ }^{-/-}$mice $\left(F_{(2,26)}=0.74, p=0.49\right)$ (Fig. $1 E$ ). Total activity levels (supplemental Table $S 1$, available at www.jneurosci.org as supplemental material) and the amplitude $\left(Q_{p} ; F_{(2,26)}=3.03, p=0.07\right)$ (Fig. $\left.1 F\right)$ of wheel-running activity in $\operatorname{Perl}^{-1}$ mice were indistinguishable from $\mathrm{Perl}^{+/+}$and Per $1^{+/-}$mice. None of the mice in our study became arrhythmic even after 3 weeks in DD.

\section{Per1-luc expression in C57BL/6J Per1 ${ }^{-/-} \mathrm{SCN}$ slices in vitro is not congruent with locomotor activity}

We next assessed Per1-luc expression in cultured SCN explanted from Per $1^{-1-}$ mice (Fig. 2). Surprisingly, we found that the rhythm of Per1-luc expression in Per1 $1^{-1-}$ whole SCN in vitro was severely affected, such that most SCN (8 of 12) were arrhythmic after a single peak (Fig. 2C). Sometimes (4 of 12) multiple, lowamplitude rhythmic components were present and significant periods were detected by $\chi^{2}$ periodogram analysis. In these cases, the period of Per1-luc expression in Per1 ${ }^{-1-}$ SCN was significantly longer than Per1 ${ }^{+/+}$, but not Per1 ${ }^{+/-}, \operatorname{SCN}\left(F_{(2,15)}=4.35\right.$, $p=0.04$, LSD $p<0.05$ ) (Fig. $2 D$ ). Since there was one peak of Per1-luc expression in Per1 ${ }^{-1-}$ SCN explants, we were able to determine the circadian phase. The phase of Per1-luc expression in $P e r 1^{-1-}$ SCN was significantly delayed by $\sim 2 \mathrm{~h}$ compared with $\operatorname{Per}^{+/+}$and Per1 ${ }^{+/-} \operatorname{SCN}\left(F_{(2,23)}=13.68, p<0.001\right.$, LSD $p<$ 0.001 ) (Fig. 2 E). The first peak of Per1-luc expression in Per $1^{-1-}$ SCN may reflect the in vivo phase, since the timing of the peak was not affected by the time of culture preparation (supplemental Fig. $\mathrm{S} 1 A$, available at www.jneurosci.org as supplemental material). The amplitude of the first cycle of Per1-luc expression in vitro in Per1 ${ }^{-1-}$ SCN explants was significantly smaller than $P e r 1^{+/-}$, but not $\operatorname{Per}^{+/+}$, SCN $(H=7.91, p=0.009$, Dunn's $p<0.05)$ (Fig. 2F). Interestingly, one arrhythmic Per1 ${ }^{-/-}$SCN explant spontaneously became rhythmic after $5 \mathrm{~d}$ in culture (supplemental Fig. S2 A, available at www.jneurosci.org as supplemental material), and another Per1 ${ }^{-1-}$ SCN slice had two to three cycles of rhythmic Per1-luc expression after a temperature pulse (supplemental Fig. S2 B, available at www.jneurosci.org as supplemental material).

We were surprised by our findings in $P e r 1^{-/-}$SCN explants because they differ markedly from a previous study (Liu et al., 2007). Liu et al. (2007) reported that PER2::LUC expression was "persistently rhythmic" in Per1 ${ }^{-1-}$ SCN explants. Furthermore, the period of PER2::LUC expression in Per1 ${ }^{-1-}$ SCN explants was similar to $P e r 1^{+/+}$SCN in their study. We next investigated the differences between our study and that of Liu et al. (2007) that could account for the contradictory results. To determine whether the use of different circadian reporters [we used Perl-luc while Liu et al. (2007) used PER2::LUC] could account for the 

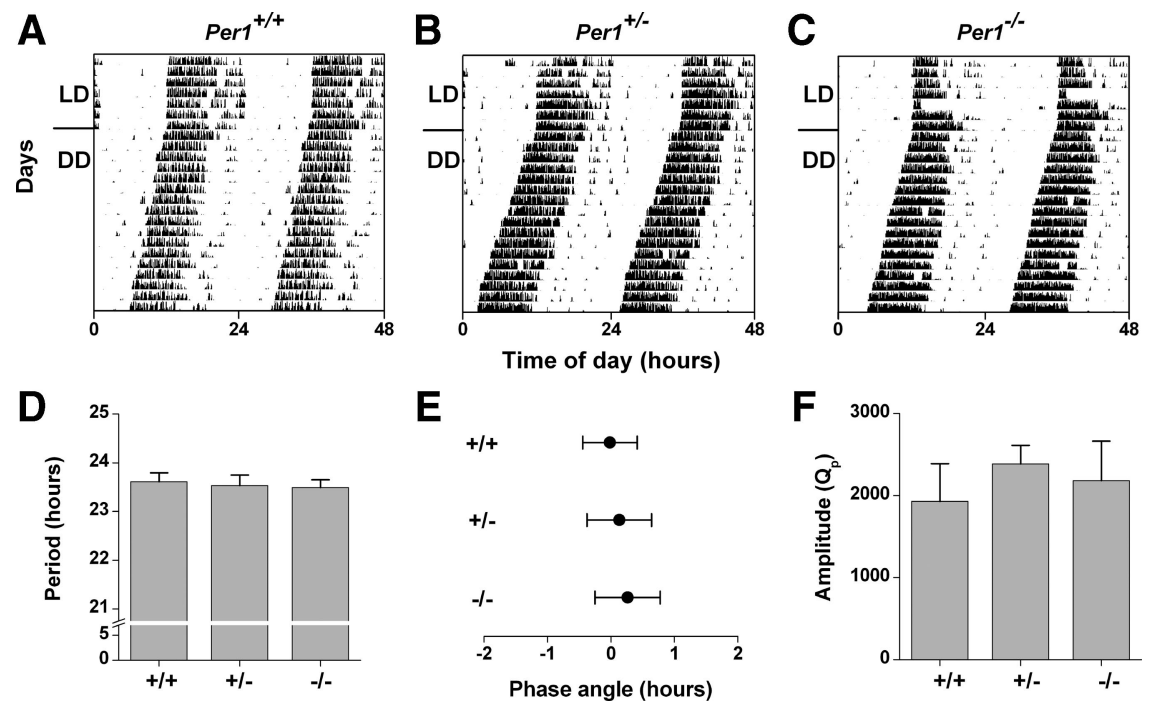

Figure 1. Circadian behavior does not differ between $\left(57 \mathrm{BL} / 6 \mathrm{~J} \operatorname{Per} 1^{+/+}, \operatorname{Per} 1^{+/-}\right.$, and $\operatorname{Per} 1^{-1-}$ mice. Representative double-plotted actograms of wheel-running activity in $\operatorname{Per}^{+/+}(\boldsymbol{A}), \operatorname{Per}^{+/-}(\boldsymbol{B})$, and $\operatorname{Per} 1^{-/-}(\boldsymbol{C})$ mice maintained in LD (lights on at $0 \mathrm{~h}$ and lights off at $12 \mathrm{~h}$ ) for $7 \mathrm{~d}$ and then released into DD. The free-running period was determined by using a $\chi^{2}$ periodogram for days $1-15$ in $D D(D)$. The phase angle of entrainment $(\boldsymbol{E})$ was determined by drawing a regression line to activity onset for days $1-5$ in $D D$ and then extending the regression line to the last day in $L D$. The phase angle was negative when activity started before lights off and positive when activity started after lights off. The amplitude $\left(Q_{p} ; \boldsymbol{F}\right)$ was the peak value of the $\chi^{2}$ periodogram. Data are the mean \pm SD.
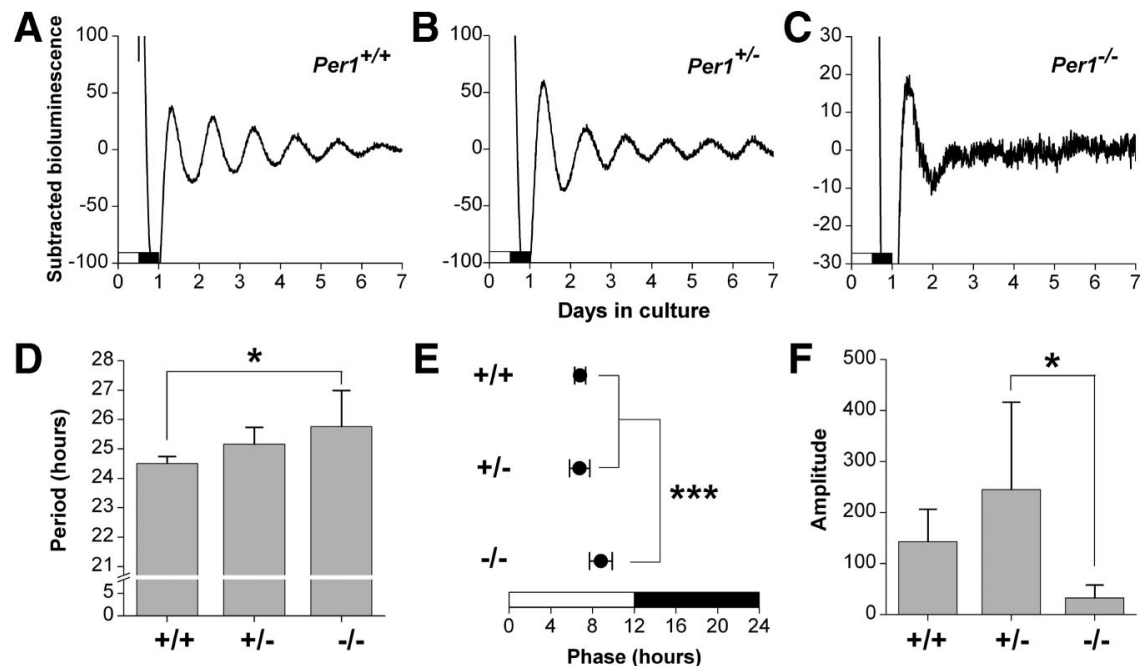

Figure 2. Per1-luc expression in SCN explants from C57BL/6J Per1 ${ }^{-1-}$ mice is arrhythmic or has low-amplitude, irregular rhythms. Representative baseline-subtracted bioluminescence rhythms in SCN explants from Per1 ${ }^{+/+}(\boldsymbol{A}), \mathrm{Per}^{1^{+-}}(\boldsymbol{B})$, and $\mathrm{Per}{ }^{-1-}(\boldsymbol{C})$ mice. The lighting condition of the previous light/dark cycle is indicated for the first day; open bars are light and black bars are dark. The scale of the $y$-axis in $(\boldsymbol{C})$ is different from in $\boldsymbol{A}$ and $\boldsymbol{B}$. $\boldsymbol{D}$, The period was determined by fitting a regression line to the acrophase of at least three cycles of the Per1-luc rhythm in all SCN except in Per1 ${ }^{-1-}$ explants, when $\chi^{2}$ periodograms were used to determine whether there was a significant period. The period of Per 1-luc expression in Per1 ${ }^{-1-} \mathrm{SCN}$ explants was determined from four $\mathrm{SCN}$ that had significant periods by $\chi^{2}$ analysis ( 8 of $12 \mathrm{SCN}$ were arrhythmic and no significant period was detected by $\chi^{2}$ analysis). One of four Per $1^{-1-}$ SCN had two significant components by $\chi^{2}$ analysis ( $24.5 \mathrm{~h}$ and $32.4 \mathrm{~h}$ ), but only the $24.5 \mathrm{~h}$ component that is in the range of circadian rhythmicity was used to calculate the mean period. $\boldsymbol{E}$, The phase was designated as the first peak of Per1-luc expression in vitro and is plotted relative to the light-dark cycle before culture, where $0 \mathrm{~h}$ is lights on and $12 \mathrm{~h}$ is lights off. $\boldsymbol{F}$, The amplitude was determined from the first cycle of Per1-luc expression in vitro. Data are the mean $\pm \mathrm{SD}^{*} p<0.05,{ }^{* * *} p<0.001$.

disparate findings, we generated $\operatorname{Per}^{-1-}$ mice carrying the PER2::LUC transgene. Consistent with our previous results, we found that PER2::LUC expression was arrhythmic or had irregular, lowamplitude rhythms in $\mathrm{Per}^{-1-}$ SCN explants (supplemental Fig. S3A, available at www.jneurosci.org as supplemental material). The lumines- cence data presented by Liu et al. (2007) showed PER2::LUC expression in Per1 ${ }^{-1-}$ SCN starting on the eighth day in culture after a media change. Therefore, we changed the media of Perl ${ }^{-1-}$ SCN explants on day 7 in vitro. We found that Perl-luc expression remained arrhythmic in Per1 $^{-1-}$ SCN explants even after a media change (supplemental Fig. S3B, available at www.jneurosci.org as supplemental material). We next prepared SCN explants of varying sizes to determine whether extraSCN tissue could confer robustness to the Per1 ${ }^{-1-}$ SCN explants. Once again, we found that circadian reporter gene expression was arrhythmic in Per1 $1^{-/-}$SCN explants regardless of their size (supplemental Fig. S3C, available at www.jneurosci.org as supplemental material).

In contrast to our contradictory results in the SCN, we found that Per1-luc expression in peripheral tissues from C57BL/6 Per1 $^{-1-}$ mice was arrhythmic (supplemental Fig. S4, available at www. jneurosci.org as supplemental material), consistent with Liu et al. (2007).

\section{Individual SCN neurons in C57BL/6J Per $^{-/-}$SCN slices have variable Per1-luc expression}

We found that Per1-luc expression was often arrhythmic in whole SCN explants from C57BL/6J Per1 ${ }^{-1-}$ mice. There are two possible explanations for this observation. First, the Per1 ${ }^{-1-}$ SCN could be composed of individual neurons that are arrhythmic. Second, it is possible that some or all of the individual SCN neurons are rhythmic, but they have widely distributed phases of Per1-luc expression. To assess these possibilities, we imaged Per1-luc luminescence in Per1 ${ }^{-1-}$ SCN explants (Fig. 3). In the absence of functional Per1, we found that some neurons exhibited robust circadian rhythmicity, while others had irregular and/or lowamplitude rhythms of Per1-luc expression (Fig. $3 H$ ). This is in contrast to Per $1^{+/+}$ SCN explants, where every cell analyzed had a robust rhythm of Per1-luc expression and the phases of individual neurons were synchronized (Fig. 3D). When bioluminescence from 10 individual neurons (Fig. $3 D$ or Fig. $3 H$ ) was averaged, the resulting trace (Fig. $3 C$ or Fig. 3G) strongly resembled the actual luminescence measured from the whole slice (Fig. $3 B$ or Fig. $3 F$ ), suggesting that the neurons analyzed faithfully represented the whole SCN.

\section{Discussion}

Surgical destruction of the SCN abolishes most circadian rhythms, including rhythmic locomotor activity (Moore and 

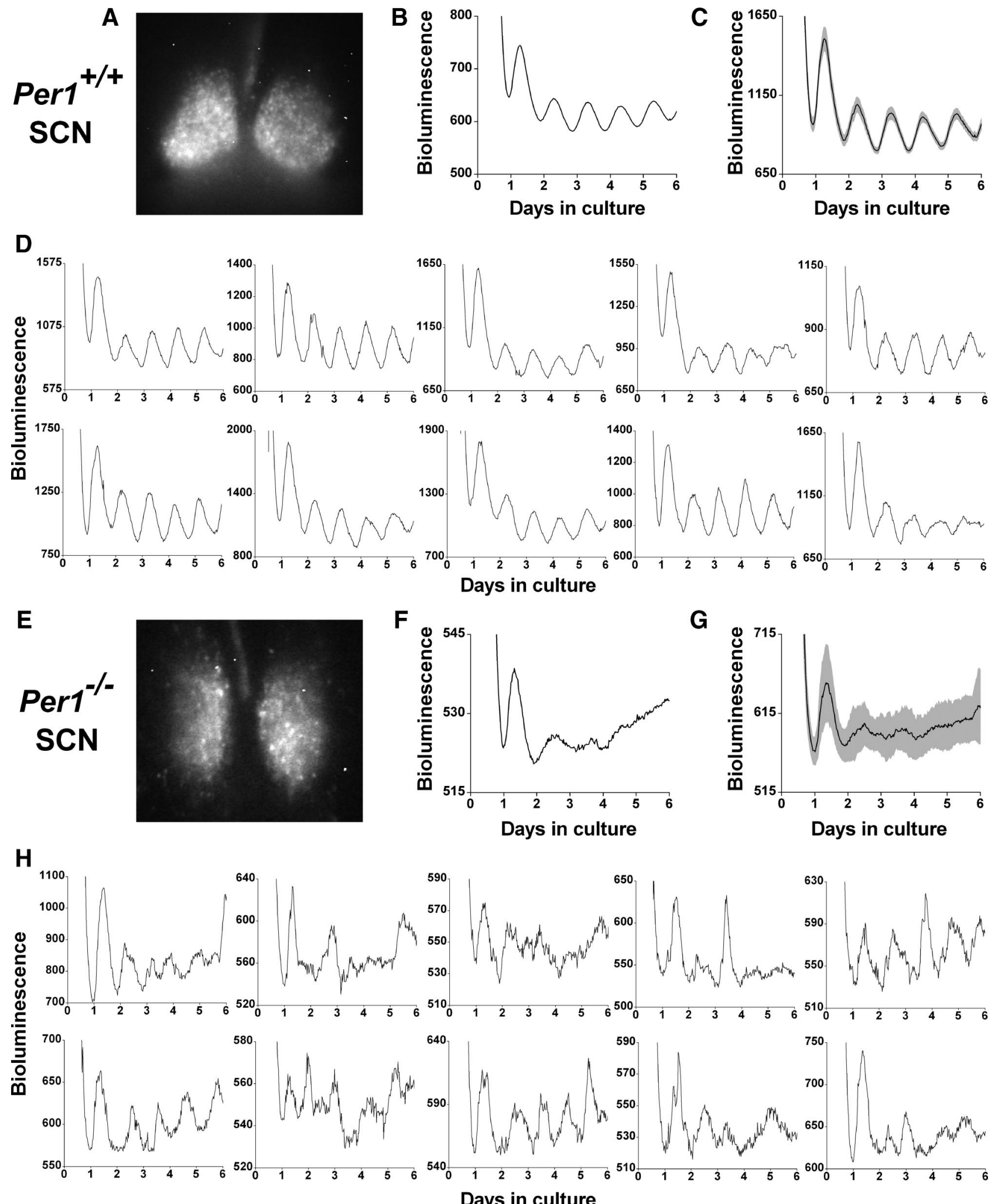

Figure 3. Imaging analysis of Per1-luc expression in SCN explanted from Per $1^{+/+}$and Per $1^{-/-}$mice. Bioluminescence (raw) was measured from the whole SCN $(\boldsymbol{A}, \boldsymbol{B}, \boldsymbol{E}, \boldsymbol{F})$ and from individual SCN neurons $(\boldsymbol{D}, \boldsymbol{H})$ in $\operatorname{Per} 1^{+/+}(\boldsymbol{A}-\boldsymbol{D})$ and $\operatorname{Per} 1^{-/-}(\boldsymbol{E}-\boldsymbol{H})$ mice. $\boldsymbol{C}, \mathbf{G}$, The mean \pm SEM (SEM shown in gray shading) of the rhythms from the 10 Per $1^{+/+}$neurons $(\boldsymbol{C})$ or from the 10 Per $1^{-/-}$ neurons $(\boldsymbol{G})$ shown in $\boldsymbol{D}$ or $\boldsymbol{H}$, respectively.

Eichler, 1972; Stephan and Zucker, 1972; Rusak and Zucker, 1979). Numerous studies suggest that the SCN encodes the period of rhythmic behavior. For example, transplanting an SCN graft into an SCN-lesioned animal restores its locomotor activity rhythm with a period identical to the donor animal (Ralph et al., 1990; Silver et al., 1996; Sujino et al., 2003), and the period of neural activity or circadian gene expression in SCN explanted from circadian mutants correlates with their free-running peri- 
ods of wheel-running activity (Liu et al., 1997, 2007; Herzog et al., 1998; Yoo et al., 2005; Meng et al., 2008). While a recent study demonstrated that disrupting coupling between SCN neurons from $\mathrm{Per} 1^{-/-}$or $\mathrm{Cry} 1^{-/-}$mice by dispersing them in culture reveals that individual cells are arrhythmic, whole SCN explants have always been found to be rhythmic if locomotor activity was rhythmic in DD (Liu et al., 2007). In contrast, we find that wheelrunning activity in C57BL/6 $\mathrm{Per} 1^{-1-}$ mice is indistinguishable from $P e r 1^{+/+}$mice, but circadian gene promoter-driven luciferase activity is arrhythmic or has an irregular, low-amplitude rhythm in whole Per $1^{-1-}$ SCN slices cultured in vitro. While we cannot account for the discrepancy between our findings and those of Liu et al. (2007), it is possible that the genetic background of the Per $1^{-1-}$ mice differed between the studies. Liu et al. (2007) used the same line of Per $1^{-1-}$ mice that we used (Bae et al., 2001), but they did not report the strain of mice used for their studies. Previous studies have demonstrated that genetic background affects circadian behavior (Ebihara et al., 1978; Possidente and Hegmann, 1982; Schwartz and Zimmerman, 1990; Shimomura et al., 2001), so it is possible that the phenotype of in vitro SCN explants could also vary among different strains of mice.

Our studies also highlight the importance of genetic background when analyzing the effect of gene knock-out on circadian behavior. Our behavioral results from $\mathrm{Per}^{-1-}$ mice on a C57BL/6J genetic background differ markedly from previous studies that examined Per $1^{-/-}$mice on mixed or isogenic 129/sv backgrounds (Bae et al., 2001; Cermakian et al., 2001; Zheng et al., 2001). We find that the period, phase angle of entrainment, total daily activity, and amplitude $\left(Q_{p}\right)$ of wheel-running activity of C57BL/6 Per1 $^{-/-}$mice do not differ from Per1 ${ }^{+/+}$mice. Additionally, in contrast to previous studies (Bae et al., 2001; Zheng et al., 2001), none of the mice in our study became arrhythmic in DD. Our data therefore suggest that the C57BL/6J genetic background can compensate for aberrant endogenous rhythms in Per $^{-1-}$ mice.

We find that circadian wheel-running behavior in C57BL/6J Per ${ }^{-/-}$mice is indistinguishable from $\mathrm{Per}^{+/+}$mice, but circadian gene promoter-driven luciferase expression in whole Per1 ${ }^{-1-}$ SCN is arrhythmic or has a low-amplitude rhythm. The low-amplitude or absent rhythm in $P e r 1^{-1-}$ SCN slices could result if the phases of individual neurons are widely dispersed or if the individual neurons are themselves arrhythmic. We find that in $P e r 1^{+/+}$SCN explants, all neurons have robust rhythms that are in phase with each other. In contrast, in SCN explants from C57BL/6 Per1 $^{-1-}$ mice, a subset of neurons maintains rhythmicity in vitro, albeit irregular, but the phases of the individual rhythms are not consolidated. These few rhythmic neurons may account for the low-amplitude rhythm of Per1-luc expression that we sometimes observe in Per1 ${ }^{-1-}$ SCN explants.

Limitations of our technique prevent us from analyzing regional differences in rhythmicity in our SCN slices. We prepare $300 \mu \mathrm{m}$ SCN slices to preserve coupling within our explants. Since we measure light emission from one focal plane, only $10-15$ cells are clearly in focus and can be analyzed for several days (resulting in fuzzy images of the SCN in Fig. 3). Therefore, there are not enough cells from each region to perform a comprehensive analysis. However, it should be noted that the cells that were analyzed were chosen without bias to a certain region and were distributed around the SCN. Future studies could assess regional differences in $\operatorname{Per} 1^{-/-}$SCN by preparing thin (50-100 $\mu \mathrm{m}$ ), sequential slices and compiling the data. For this study, we preferred to keep coupling intact in $P e r 1^{-1-}$ SCN to avoid any factors that could account for the low-amplitude, irregular rhythms that we observed.

We also find that the Per1-luc rhythm can be initiated in Per $1^{-1-}$ SCN explants when they are exposed to a temperature change (e.g., opening the incubator that contains the LumiCycle). A previous study demonstrated that a heat shock induced luciferase expression in PER2::LUC-expressing liver and lung explants (Kornmann et al., 2007). It is possible that the phases of the small subset of rhythmic neurons in $\mathrm{Per}^{-1-} \mathrm{SCN}$ are synchronized following a temperature pulse.

Together, our data raise two critical questions regarding timekeeping by the mammalian circadian system. First, do circadian rhythms measured from ex vivo SCN and peripheral tissues accurately reflect the in vivo phenotype of the tissue? While mutant phenotypes may be readily revealed in vitro, our data suggest that in vivo factors, such as coupling with extra-SCN oscillators or feedback to the SCN, may compensate for the aberrant endogenous rhythms of mutant SCN. Second, is a small subset of rhythmic neurons adequate to drive behavior? A previous study showed that only a few vasoactive intestinal peptide-positive cells were present in grafts of cell suspensions from donor SCN that restored rhythmic locomotor behavior to SCN-lesioned hamsters (Silver et al., 1990). These data suggest that only a small population of SCN neurons may be necessary to produce behavioral rhythms. Since we find that only a few individual neurons in Per $1^{-1-}$ SCN explants have robust $24 \mathrm{~h}$ rhythms, it is possible that the ensemble of neurons in the SCN is not necessary to control behavior, but rather a small number of rhythmic neurons are sufficient to regulate wheel-running activity. Further analysis of the in vivo phenotype of C57BL/6 Perl $^{-1-}$ SCN is necessary to explore these issues.

\section{References}

Aton SJ, Block GD, Tei H, Yamazaki S, Herzog ED (2004) Plasticity of circadian behavior and the suprachiasmatic nucleus following exposure to non-24-hour light cycles. J Biol Rhythms 19:198-207.

Bae K, Jin X, Maywood ES, Hastings MH, Reppert SM, Weaver DR (2001) Differential functions of mPer1, mPer2, and mPer3 in the SCN circadian clock. Neuron 30:525-536.

Cermakian N, Monaco L, Pando MP, Dierich A, Sassone-Corsi P (2001) Altered behavioral rhythms and clock gene expression in mice with a targeted mutation in the Period1 gene. EMBO J 20:3967-3974.

Ebihara S, Tsuji K, Kondo K (1978) Strain differences of the mouse's freerunning circadian rhythm in continuous darkness. Physiol Behav 20:795-799.

Herzog ED, Takahashi JS, Block GD (1998) Clock controls circadian period in isolated suprachiasmatic nucleus neurons. Nat Neurosci 1:708-713.

Herzog ED, Aton SJ, Numano R, Sakaki Y, Tei H (2004) Temporal precision in the mammalian circadian system: a reliable clock from less reliable neurons. J Biol Rhythms 19:35-46.

Ko CH, Takahashi JS (2006) Molecular components of the mammalian circadian clock. Hum Mol Genet 15:R271-R277.

Kornmann B, Schaad O, Bujard H, Takahashi JS, Schibler U (2007) Systemdriven and oscillator-dependent circadian transcription in mice with a conditionally active liver clock. PLoS Biol 5:e34.

Liu AC, Welsh DK, Ko CH, Tran HG, Zhang EE, Priest AA, Buhr ED, Singer O, Meeker K, Verma IM, Doyle FJ 3rd, Takahashi JS, Kay SA (2007) Intercellular coupling confers robustness against mutations in the SCN circadian clock network. Cell 129:605-616.

Liu C, Weaver DR, Strogatz SH, Reppert SM (1997) Cellular construction of a circadian clock: period determination in the suprachiasmatic nuclei. Cell 91:855-860.

Meng QJ, Logunova L, Maywood ES, Gallego M, Lebiecki J, Brown TM, Sládek M, Semikhodskii AS, Glossop NR, Piggins HD, Chesham JE, Bechtold DA, Yoo SH, Takahashi JS, Virshup DM, Boot-Handford RP, Hastings MH, Loudon AS (2008) Setting clock speed in mammals: the CK1 epsilon tau mutation in mice accelerates circadian pacemakers by selectively destabilizing PERIOD proteins. Neuron 58:78-88. 
Molyneux PC, Dahlgren MK, Harrington ME (2008) Circadian entrainment aftereffects in suprachiasmatic nuclei and peripheral tissues in vitro. Brain Res 1228:127-134.

Moore RY, Eichler VB (1972) Loss of a circadian adrenal corticosterone rhythm following suprachiasmatic lesions in the rat. Brain Res 42: 201-206.

Possidente B, Hegmann JP (1982) Gene differences modify Aschoff's rule in mice. Physiol Behav 28:199-200.

Ralph MR, Foster RG, Davis FC, Menaker M (1990) Transplanted suprachiasmatic nucleus determines circadian period. Science 247:975-978.

Rusak B, Zucker I (1979) Neural regulation of circadian rhythms. Physiol Rev 59:449-526.

Schwartz WJ, Zimmerman P (1990) Circadian timekeeping in BALB/c and C57BL/6 inbred mouse strains. J Neurosci 10:3685-3694.

Shimomura K, Low-Zeddies SS, King DP, Steeves TD, Whiteley A, Kushla J, Zemenides PD, Lin A, Vitaterna MH, Churchill GA, Takahashi JS (2001) Genome-wide epistatic interaction analysis reveals complex genetic determinants of circadian behavior in mice. Genome Res 11:959-980.

Silver R, Lehman MN, Gibson M, Gladstone WR, Bittman EL (1990) Dispersed cell suspensions of fetal SCN restore circadian rhythmicity in SCNlesioned adult hamsters. Brain Res 525:45-58.

Silver R, LeSauter J, Tresco PA, Lehman MN (1996) A diffusible coupling signal from the transplanted suprachiasmatic nucleus controlling circadian locomotor rhythms. Nature 382:810-813.
Stephan FK, Zucker I (1972) Circadian rhythms in drinking behavior and locomotor activity of rats are eliminated by hypothalamic lesions. Proc Natl Acad Sci U S A 69:1583-1586.

Sujino M, Masumoto KH, Yamaguchi S, van der Horst GT, Okamura H, Inouye ST (2003) Suprachiasmatic nucleus grafts restore circadian behavioral rhythms of genetically arrhythmic mice. Curr Biol 13:664-668.

Takahashi JS, Turek FW, Moore RY (2001) Circadian clocks. New York: Kluwer Academic/Plenum.

Yamazaki S, Takahashi JS (2005) Real-time luminescence reporting of circadian gene expression in mammals. Methods Enzymol 393:288-301.

Yoo SH, Yamazaki S, Lowrey PL, Shimomura K, Ko CH, Buhr ED, Siepka SM, Hong HK, Oh WJ, Yoo OJ, Menaker M, Takahashi JS (2004) PERIOD2::LUCIFERASE real-time reporting of circadian dynamics reveals persistent circadian oscillations in mouse peripheral tissues. Proc Natl Acad Sci U S A 101:5339-5346.

Yoo SH, Ko CH, Lowrey PL, Buhr ED, Song EJ, Chang S, Yoo OJ, Yamazaki S, Lee C, Takahashi JS (2005) A noncanonical E-box enhancer drives mouse Period2 circadian oscillations in vivo. Proc Natl Acad Sci U S A 102:2608-2613.

Zheng B, Albrecht U, Kaasik K, Sage M, Lu W, Vaishnav S, Li Q, Sun ZS, Eichele G, Bradley A, Lee CC (2001) Nonredundant roles of the mPer1 and mPer2 genes in the mammalian circadian clock. Cell 105: 683-694. 